\title{
Impact of the COVID-19 pandemic on the trauma and orthopaedic department at level one Major Trauma Centre in the republic of Ireland
}

\author{
Hany Elbardesy, Eoghan Meagher, Shane Guerin
}

From the Department of trauma and orthopaedic, Cork University Hospital, Cork, Ireland

\begin{abstract}
The Coronavirus Disease (COVID-19) has been identified as the cause of a rapidly spreading respiratory illness in Wuhan, Hubei Province, China in early December 2019. Since then, the free movement of people has decreased. The trauma-related injuries and the demand on the trauma and orthopaedic service would be expected to fall. The aim of this study to examine the impact of the COVID-19 pandemic on a level 1 Trauma Centre in the Republic of Ireland (ROI). Patients admitted to the Trauma \& Orthopaedic (T\&O) Department at Cork University Hospital (CUH) and the South Infirmary Victoria University Hospital (SIVUH), and their associated fracture patterns and management, between 01/03/20 and the 15/04/20 were documented and compared to the patient admissions from the same time period one year earlier in 2019. The total number of T\&O operations performed decreased by $10.15 \%(\mathrm{P}=$ 0.03)between the two time periods. The number of paediatric procedures fell by $40.32 \%(\mathrm{P}=0.15)$. Adult Distal radius and paediatric elbow fractures (excluding supracondylar fracture) increased by $88 \%$ and $13 \%(P=0.19),(P=0.04)$ respectively. Hip fractures remained the most common fracture-type admitted for surgery. The COVID-19 crisis has to lead to a decrease in the total numbers of trauma surgeries in a major trauma centre in the ROI. This decline is most evident in the number of paediatric and male adult patients presenting with fractures requiring operative management. Interestingly, fractures directly related to solo outdoor activities, such as running or cycling, as well as simple mechanical falls
\end{abstract}

The authors declare that they have no conflict of interest. There is no funding source.

This article does not contain any studies with human participants or animals performed by any of the authors. like ankle, distal radius, elbow, and hand fractures all increased. Irish males were more compliant with outdoors restrictions than females.

Keywords : COVID-19 ; pandemic ; trauma ; orthopaedics ; surgery ; Ireland.

\section{INTRODUCTION}

The COVID-19 pandemic is a catastrophic global healthcare crisis that started in Wuhan, China, in late 2019 (2,36), and has spread at an exponential rate worldwide (1), extending as far as the United States (19) Australia (30) and Italy (18). The World Health Organization (WHO) identified this new coronavirus outbreak as a global health emergency, seeking international help in an effort to control this epidemic (10). Healthcare systems have been stretched physically, financially, and mentally in an effort to curtail this crisis with damage-limitation

Hany Elbardesy, MSc, MRCS, FRCSeng, FEBOT,

Eoghan Meagher, MRCS

- Shane Guerin, FRCS

Department of trauma and orthopaedic, Cork University Hospital, Cork, Ireland.

Correspondence: Hany Elbardesy, Department of trauma and orthopaedic, Cork University Hospital, Cork, Ireland. Phone: +353892106657, +353214347890.

Email : elbardecy@hotmail.com

- 2021, Acta Orthopædica Belgica. 
at the forefront of these measures. These efforts declared themselves in many different ways, from redeploying healthcare staff (22) to building new temporary hospitals in days and weeks (4). Most countries have introduced compulsory partial or total lockdown. The Irish Government introduced mandatory restrictions to outdoor activity in an effort to deter the spread of COVID-19 from March $1^{\text {st }} 2020$. This was followed by total lockdown of the country which was introduced at the end of March 2020 with the initial lockdown period intended for 2 weeks but this was extended to the end of May 2020. Total lockdown consisted of the closure of schools and universities, all factories, most workplaces not deemed essential, and a complete ban on social/family gatherings in houses or gardens. The entire population was asked to restrict movement to within a $5 \mathrm{~km}$ radius of their homes and stay at home. Weddings were limited to 25 people, and bars/restaurants can serve takeaway food/beverages exclusively until the end of 2020 (32). The number of people outdoors decreased, and with it, the number of accidents related to outdoor-activities also declined with high rate of restrictions obligation among people. The Royal College of Surgeons of Ireland (RCSI) published new guidance for surgical prioritisation. It classified Surgeries into four categories according to the level of urgency. Priority level 1a refers to an emergency that needs surgery within 24 hours. Priority level $1 \mathrm{~b}$ is for the urgent operation needed with 72 hours. Priority level 2 is for surgery that can be deferred for up to 4 weeks. Priority level 3 Surgery can be delayed for up to 3 months and priority level 4 refers to surgery that can be delayed for more than three months (31). Consequently, all elective cases have been postponed to limit the demand on health-care resources and to keep the hospital beds available, if needed, for emergency admissions. Other countries such as Singapore, Australia and New Zealand employed different approaches with the same principles that were based on clinical urgency, patient and health-care worker protection and conservation of health-care resources $(22,33)$. The relationship between this COVID-19 epidemic and Orthopaedic surgery appears disparate when compared with other medical specialities such as
Infectious Diseases, Emergency Medicine, and Internal Medicine. However, as part of the larger health-care ecosystem, Orthopaedic surgeons also have a role in this pandemic. This study was conducted to measure the effect this crisis was having on a level one Major Trauma Centre in the Republic of Ireland (ROI).

\section{PATIENTS AND METHODS}

This is an observational study examining the number of patients admitted to the Trauma and Orthopaedic (T\&O) department at Cork University Hospital (CUH) and the South Infirmary Victoria University Hospital (SIVUH) over a 6-weeks period between the $01 / 03 / 20$ and $15 / 04 / 20$. Our inclusion criteria were any patient presented to the emergency department (ED) of the $\mathrm{CUH}$ and required surgical intervention by the trauma and orthopaedics department's team. Our exclusion criteria were all trauma patients that were treated conservatively. Patient fracture types and management patterns were documented and subsequently compared to the patient data from the same time window in 2019.

CUH is a level 1 trauma centre, it is the only hospital in Cork that manages T\&O. The city of Cork covers a total surface area of 820 square kilometres in the south of ROI. The population density is approximately 3,300 individuals per square kilometre in the city, and this density drops to about 480 per square kilometre when looking at the metropolitan area. The current population is 417,211 , with 206,953 males and 210,258 females (9). Typically, pre-pandemic, all trauma surgeries were performed in two operating rooms (OR) at CUH daily except at weekends and on public holidays; during which only one OR was available. SIVUH was used solely for elective cases. Since the advent of this crisis, CUH has but one dedicated OR for T\&O surgery that is kept exclusively for elderly medically unwell patients, mostly accounted for by hip fracture patients. The reasoning behind this approach was based on the literature documenting the fact that older patients with medical co-morbidities are more adversely affected by COVID-19 infection owing to their low functional reserves and weakened immune systems 
$(11,30,35)$. Two ORs at the SIVUH have been made available for all other trauma cases; all electives surgeries were postponed. In the Trauma \& Orthopaedic Department. there was a shift towards more conservative management of some fractures, where at all possible, especially distal radius fractures in keeping with international guidelines $(13,14)$. Owing to COVID-19 restrictions imposed by Infection Prevention Control and Anaesthetic teams, perioperative times also increased.

Demographic data, medical history, and clinical and radiological data at presentation were collected.

Statistical analysis was performed using the software package Statistical Package for the Social Sciences (SPSS) Version 26.0.0 (SPSS Inc., Chicago, IL, USA) (28). To determine the statistical significance of a test a $\mathrm{P}$ value of $\leq 0.05$ was set (34). Continuous variables are presented as mean with standard deviation; categorical variables are presented as count (percent). Independent twosample $t$ test and Chi-square test were used as appropriate to compare groups.

\section{RESULTS}

In total, 505 patients across the two time periods requiring acute surgery were included. The total number of operations decreased by $10.15 \%$, from 266 to 239 in the same time period last year. This was a statistically significant difference $(\mathrm{P}=0.03)$. No significant changes in the total number of patients between March 2019 and 2020 (172 and 174 respectively), however, the main changes were between April 2019 and 2020, (94 and 65 respectively). The percentage of reduction was $30.85 \%$, $(\mathrm{P}=0.11)$. Interestingly, some specific operation numbers increased, such as distal radius fracture in the adult population. The incidence of these rose from 18 to 34 , reflecting an $88.88 \%$ increase $(P=0.19)$. In addition to these fractures in the adult population, A breakdown of operative numbers and population data can be found in Table 1 and (Fig 1 and 2).

The females to male ratio were changed from 147 and 119 in 2019 to 147 and 92 in 2020 . The reduction was only among the male population $(22.69 \%)$. The principal reduction in the numbers of males was mainly in April 2020 from 45 to 21 (53.34\%), P = 0.11 , which reflects that the Irish males were more compliant with outdoors restrictions than females.

In the period from 1st of March to the $15^{\text {th }}$ of April 2019 , the total numbers of patients with hip fractures were 66, 43 females and 23 males. The mean age was $77.9 \pm 10.5$ years. For the same period in 2020,

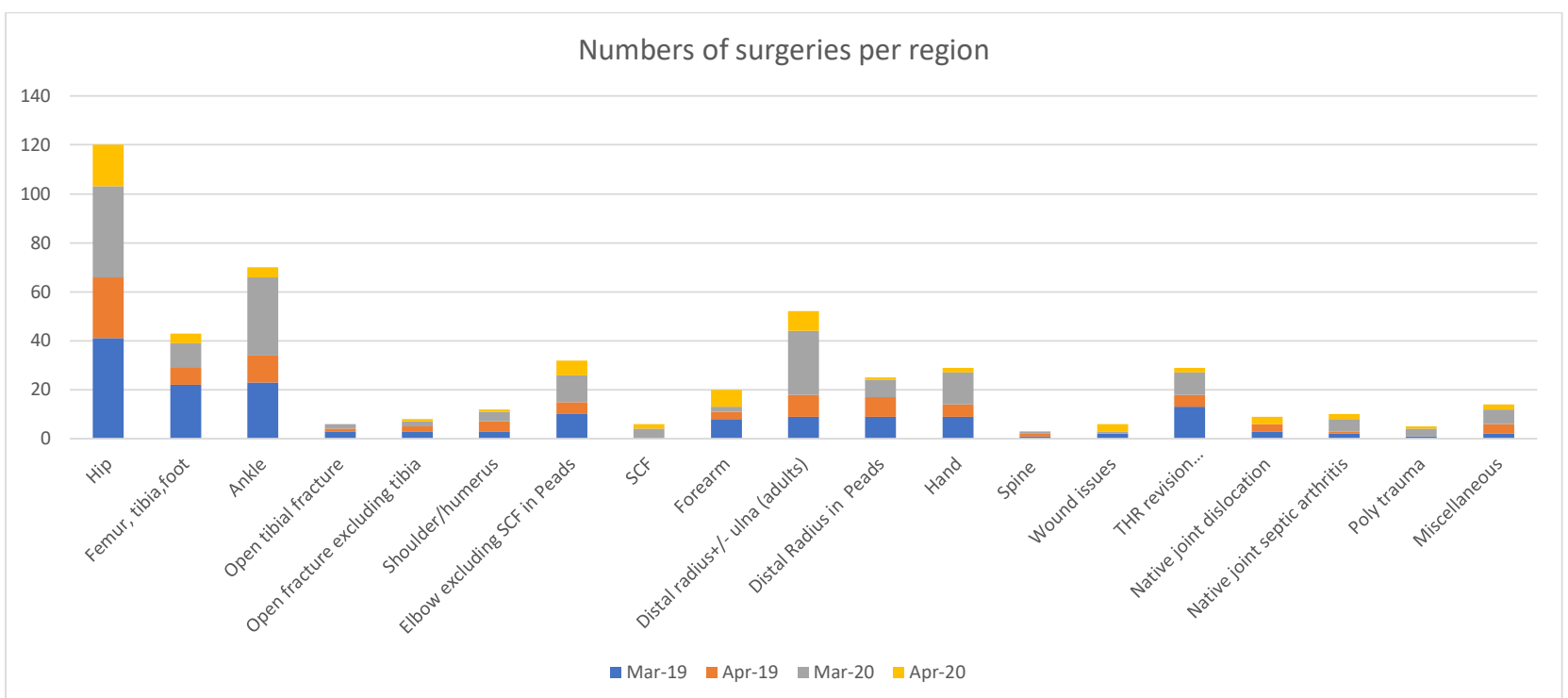

Figure 1. - The total numbers of surgeries per region with comparison between the March 2019 and 2020 and the first half of April 2019 and 2020, SCF(supracondylar fracture), Peads (paediatrics). 







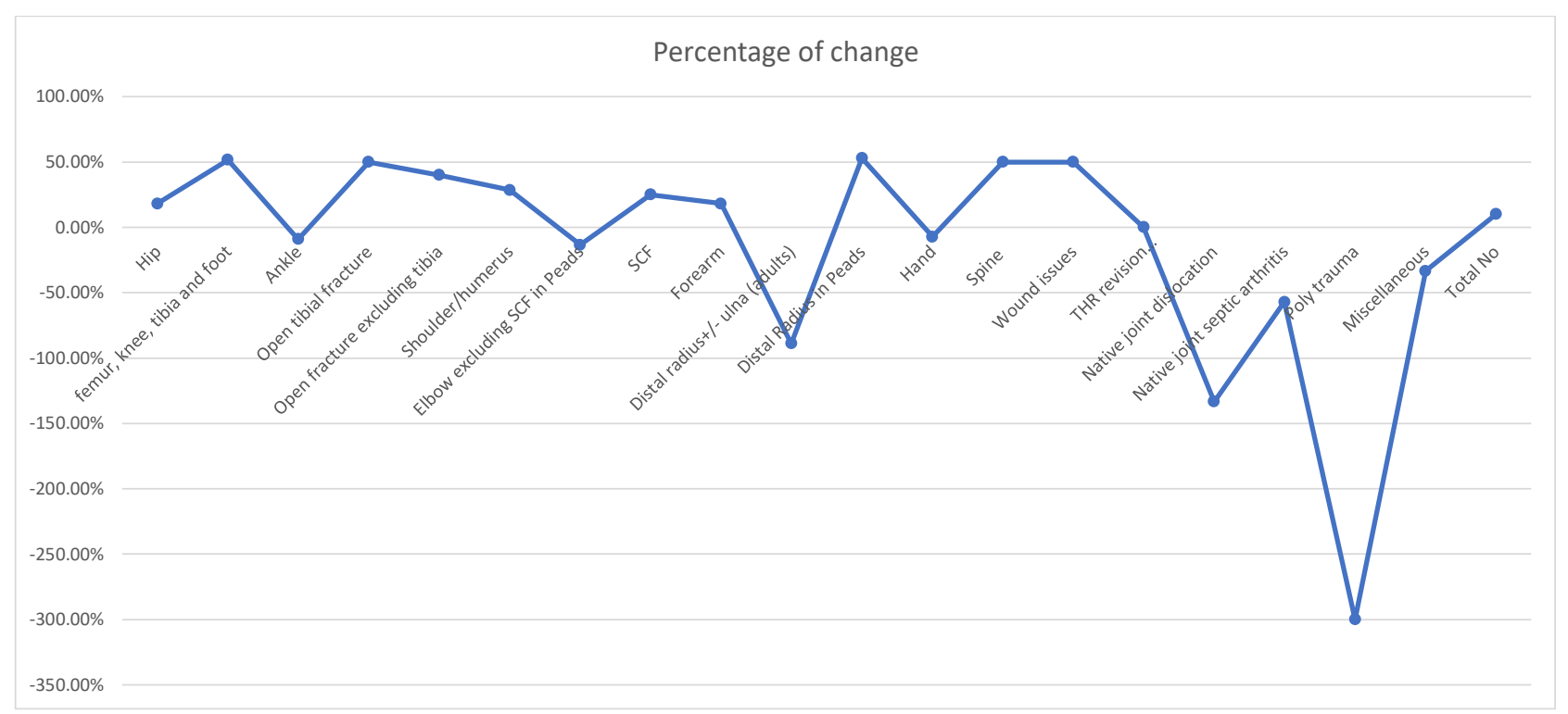

Figure 2. - The percentage of reduction of numbers of surgery.

the total numbers of hip fractures dropped to 54 , 38 females and 16 males, the mean age was $77.9 \pm$ 16.5. The overall reduction was $18.18 \%(\mathrm{P}=0.06)$. Of note, the decline was mainly in the men patients $(30.43 \%)$ from 23 to 16 . In comparison to the females that show a sort of stability, the reduction percentage was only $11.6 \%$, from 43 to 38 .

The number of operations performed on children fell from 62 to 37, denoting a 40.32\% reduction $(\mathrm{P}=$ $0.15)$. However, paediatric elbow fractures without supracondylar fracture (SCF) also increased from 15 to 17 ; a rise of $13.33 \%$ from the year earlier $(\mathrm{P}=$ 0.04).The SCF was decreased by $25 \%$ from 8 to 6 $(\mathrm{P}=0.09)$. The distal radius fractures dropped nearly to half ,52.93 from 17 to $8(\mathrm{p}=0.2)$

The total numbers of lower limb trauma that included fracture femur, knee, tibia, ankle and foot fell from 66 (41 females and 25 male) to 52 (36 females and 16 male). Notice worthy, there were $36 \%$ reduction in the number of male (from 25 to 16$),(\mathrm{P}=0.1)$, in comparison to $12.19 \%$ only in the female population ( from 41 to 36$),(\mathrm{P}=0.4)$. After exclusion of ankle and open fractures, the remaining lower limb injuries dropped significantly by $51,72 \%$ from 29 to $14,(\mathrm{P}=0.2)$. Also most of reductions were among the men (from 13 to 4 ), that represents $69.23 \%$, however, only $37.5 \%$. In contrast to the ankle fracture that slightly increased by $9.09 \%$ from 33 to $36(\mathrm{P}=0.2)$. Additionally, the open tibial fracture showed non-significant drop from 4 to $2,(\mathrm{P}=0.2)$.

The total number of adult's trauma was surprisingly increased by 23.07 from 65 (31 women and 34 men) to 80 (47 women and 33 men ), ( $\mathrm{P}=$ 0.6).all the increase was among ladies while the number of males slightly decreased from 34 to 33. The elbow and hand slightly increased from 15 and 14 to 17 and 15 respectively, however the distal radius fracture almost doubled from 18 to 34 ( $\mathrm{P}=$ $0.1)$.

\section{DISCUSSION}

Trauma and Orthopaedic practice worldwide has been markedly affected by this viral outbreak. In Ireland, the RCSI has postponed all elective surgeries to allow hospitals to keep beds for the treatment of patients with confirmed or suspected COVID-19 (31). In contrast, hospitals in Singapore have kept both orthopaedic oncology surgeries and surgical day cases (requiring $\leq 23$ hours of hospital stay). These include arthroscopies (knees, shoulders and ankles) and simple day procedures such as soft-tissue surgical procedures and elective implant removals (10) . 
Some authors suggest the implementation of novel technologies such as wearable sensors $(12,27)$ and the use of videoconferencing (25) tools to monitor patient outcomes remotely (e.g., knee range of motion after knee arthroplasty) to minimise the numbers of patients presenting to the hospital (10). We have successfully used technology-assisted rehabilitation (e.g., online educational platforms, video assisted physiotherapy and rehabilitation ) that demonstrated a significant improvement in patient satisfaction, pain, and outcome scores $(20,21,26)$.

Before this crisis, total compliance in CUH with guidelines such as the British Orthopaedic Association Standards for Trauma (BOAST) and the National Institute for Health and Care Excellence (NICE) guidelines ultimately could not be achieved, especially regarding the time to surgery in ankle and hip fracture patients. This failure to comply with the a fore mentioned guidelines was attributed to the high number of patients requiring surgery daily which usually exceeded the capacity of two ORs. According to BOAST for ankle fractures and NICE guidelines for hip fractures, patients should have surgery the same day or the following day $(23,29)$. Since the advent of COVID-19, and the decision to postpone electives cases, which has directly lead to an increased capacity facilitated by the availability of three T\&O ORs, waiting times have dramatically decreased, and compliance with these guidelines is much improved.

The COVID-19 pandemic has impacted the numbers of T\&O surgeries performed in a level 1 Major Trauma Centre in the south of the ROI but not to a massive extent. While statistically significant, only a $10.15 \%$ decline between the two time periods was observed $(\mathrm{P}-\mathrm{value}=0.03)$. Interestingly, and perhaps expected, the number of operations performed on children decreased by $40 \%$. School closures and government-mandated cessation of all outdoor group activities, organised sports and decreased playground use (32,7). likely explain this decline in paediatric fracture presentations. The most common reason for supracondylar fractures, which have fallen by $25 \%$ in this observational study, are falls from playground equipment and falls from a standing height $(7,24)$.
Individual/solo sporting activities like running and cycling were not prohibited which are the main reasons for fractures of the ankle, elbow, hand and distal radius $(6,7)$. In this study, the number of these fractures increased by $9.09 \%, 13.33 \%, 7.14 \%$, and $88.88 \%$, respectively. Of note, the numbers of cycling-related major trauma in Ireland has been growing in recent years $(8,17)$. The number of long bone fractures such as humerus, femur and tibia fractures documented also fell; possibly because these fracture types usually require high energy trauma (5), accounted for mostly by Road Traffic Accidents (3). There was also a reduction in hip fractures by $18.18 \%(\mathrm{P}=0.06)$, but these remained the most common fracture type warranting surgery (16).

Interestingly, the number of females requiring T\&O Surgery between the two time periods observed was precisely the same. Any reduction in overall numbers was accounted for by the fall in the number of adult male patients only, down from 119 to 92 denoting a $22.69 \%$ decrease ( $\mathrm{P}$-value $=0.08$ ). The Irish incidence of male hip fractures is less than that of females (16). which is consistent with the hip fracture patterns worldwide (15).

The authors acknowledge that a limitation of this study is the collection of data from one centre only. Multi-centre studies with larger cohorts are warranted to add to the growing body of literature documenting the impact of COVID-19 on T\&O surgery not only locally, but internationally.

\section{CONCLUSION}

The COVID-19 crisis has led to a decrease in the total numbers of trauma surgeries in a level one Major Trauma Centre in the ROI when compared to the same time period one year prior. This reduction is a result of the decline in the number of paediatric and male adult patients presenting. However, fractures directly related to individual/solo outdoor activities such as running or cycling, and fractures as a result of mechanical falls such as ankle, distal radius, elbow, and hand fractures demonstrably increased. 


\section{REFERENCES}

1. (WHO) WHO. Coronavirus disease 2019 (COVID-19) Situation Report. Data as Rep by Natl Authorities by 1000 CET 31 March 20202020 ; 2019 : Data as reported by national authorities by 10:00.

2. Adhikari SP, Meng S, Wu Y-J, et al. Epidemiology, causes, clinical manifestation and diagnosis, prevention and control of coronavirus disease (COVID-19) during the early outbreak period : a scoping review. Infect Dis Poverty $2020 ; 9: 29$.

3. Ailbhe Jordan. Coronavirus Ireland news : Restrictions improve air quality in Dublin and Cork's worst traffic blackspots. https:/WwwIrishmirrorIe/All-about/RoadTraffic-Accidents 2020.

4. Ashford RU, Nichols JS, Mangwani J. Annotation : The COVID-19 Pandemic and Clinical Orthopaedic and Trauma Surgery. J Clin Orthop Trauma 2020.

5. Bandyopadhyay O, Biswas A, Bhattacharya BB. Longbone fracture detection in digital X-ray images based on digital-geometric techniques. Comput Methods Programs Biomed 2016; $123: 2-14$.

6. Bonazza N, Smuin DM, Sterling N, et al. Epidemiology of Surgical Treatment of Adolescent Sports Injuries in the United States : Analysis of the MarketScan Commercial Claims and Encounters Database. Arthrosc Sport Med Rehabil 2019; 1 : e59-e65.

7. Bram JT, Johnson MA, Magee LC, et al. Where Have All the Fractures Gone? The Epidemiology of Pediatric Fractures During the COVID-19 Pandemic. J Pediatr Orthop 2020 ; Publish Ah.

8. Broe MP, Kelly JC, Groarke PJ, et al. Cycling and spinal trauma: A worrying trend in referrals to a national spine centre. Surg $2018 ; 16: 202-206$.

9. Census. Census 2016 Reports. 2016.

10. Chang Liang Z, Wang W, Murphy D, et al. Novel Coronavirus and Orthopaedic Surgery : Early Experiences from Singapore. J Bone Joint Surg Am. 2020.

11. Chen N, Zhou M, Dong $X$, et al. Epidemiological and clinical characteristics of 99 cases of 2019 novel coronavirus pneumonia in Wuhan, China : a descriptive study. Lancet $2020 ; 395: 507-513$.

12. Chiang C-Y, Chen K-H, Liu K-C, et al. Data Collection and Analysis Using Wearable Sensors for Monitoring Knee Range of Motion after Total Knee Arthroplasty. Sensors $2017 ; 17: 418$.

13. Chung KC, Kim HM, Malay S, et al. The Wrist and Radius Injury Surgical Trial : 12-Month Outcomes from a Multicenter International Randomized Clinical Trial. Plast Reconstr Surg 2020 ; 145 : 1054e-1066e.

14. Costa ML, Achten J, Rangan A, et al. Percutaneous fixation with Kirschner wires versus volar locking-plate fixation in adults with dorsally displaced fracture of distal radius : five-year follow-up of a randomized controlled trial. Bone Joint J 2019 ; 101-B : 978-983.
15. Dhanwal D, Dennison E, Harvey $\mathbf{N}$, et al. Epidemiology of hip fracture : Worldwide geographic variation. Indian $J$ Orthop $2011 ; 45: 15$.

16. Dodds MK, Codd MB, Looney A, et al. Incidence of hip fracture in the Republic of Ireland and future projections : a population-based study. Osteoporos Int 2009; 20 : 21052110.

17. Foley J, Cronin M, Brent L, et al. Cycling related major trauma in Ireland. Injury $2020 ; 51: 1158-1163$.

18. Giovanetti $M$, Benvenuto $D$, Angeletti $S$, et al. The first two cases of 2019-nCoV in Italy: Where they come from? J Med Virol 2020 ; 92 : 518-521.

19. Holshue ML, DeBolt $C$, Lindquist $S$, et al. First Case of 2019 Novel Coronavirus in the United States. N Engl J Med $2020 ; 382$ : 929-936.

20. Koo K, Park DK, Youm YS, et al. Enhanced Reality Showing Long-Lasting Analgesia after Total Knee Arthroplasty : Prospective, Randomized Clinical Trial. Sci Rep $2018 ; 8: 2343$.

21. Lin F, Chen M, Li P. Influence of structured telephone follow-up on patient compliance with rehabilitation after total knee arthroplasty. Patient Prefer Adherence 2016 : 257.

22. Murphy ZCL. Novel Coronavirus and Orthopaedic Surgery Early Experiences from SingaporeNo Title. J Bone Jt Surg Am 2020 ; Mar 20 : e.

23. NICE. Hip fracture : management acture : management Clinical guideline. Natl Inst Heal Care Excell 2011 : 32.

24. Pilla NI, Rinaldi J, Hatch M, et al. Epidemiological Analysis of Displaced Supracondylar Fractures. Cureus 2020.

25. Piqueras M, Marco E, Coll M, et al. Effectiveness of an interactive virtual telerehabilitation system in patients after total knee arthoplasty: A randomized controlled trial. $J$ Rehabil Med 2013 ; 45 : 392-396.

26. Russell TG, Buttrum P, Wootton R, et al. Internet-Based Outpatient Telerehabilitation for Patients Following Total Knee Arthroplasty. J Bone Jt Surgery-American Vol 2011 ; $93: 113-120$.

27. Small SR, Bullock GS, Khalid S, et al. Current clinical utilisation of wearable motion sensors for the assessment of outcome following knee arthroplasty : a scoping review. BMJ Open 2019 ; 9 : e033832.

28. SPSS Inc, Chicago, IL U. IBM SPSS Statistics 2019.

29. Strauss EJ, Egol KA. The management of ankle fractures in the elderly. Injury $2007 ; 38: 2-9$.

30. Team 2019-nCoV National Incident Room Surveillance. 2019-nCoV acute respiratory disease. Aust Epidemiol Rep 1 (Reporting Week 26 January-1 Febr 2020) n.d. ; 6 : 44-44.

31. The Royal College of Surgeons of England ; Royal college of physicians and surgeons of Glasgow; RCSI; Royal College of Surgeons of Edinburgh. Clinical guide to surgical prioritisation during the coronavirus pandemic 2020.

32. Theguardiancom. 'Stay home': Varadkar announces sweeping two-week lockdown. 'Stay Home': Varadkar 
Announces Sweeping Two-Week Lockdown. n.d. https:// www.theguardian.com/world/2020/mar/27/stay-homevaradkar-urges-irish-in-drastic-lockdown.

33. Varghese $\mathbf{C}, \mathbf{X u ~ W}$. Quantifying what could have been - the impact of the Australian and New Zealand governments' response to COVID-19. Infect Dis Heal 2020.

34. Yazici H. The P-value crisis and the issue of causality. Rheumatology 2020.
35. Zhou F, Yu T, Du R, et al. Clinical course and risk factors for mortality of adult inpatients with COVID-19 in Wuhan, China : a retrospective cohort study. Lancet 2020 ; 395 : 1054-1062.

36. Zhu N, Zhang D, Wang W, et al. A Novel Coronavirus from Patients with Pneumonia in China, 2019. N Engl $J$ Med $2020 ; 382: 727-733$. 